\title{
SECOND ORDER DEHN FUNCTIONS AND HNN-EXTENSIONS
}

\author{
X. WANG and S. J. PRIDE \\ Dedicated to M. F. (Mike) Newman on the occasion of his 65th birthday
}

(Received 25 January 1999; revised 27 May 1999)

Communicated by E. A. O'Brien

\begin{abstract}
In previous work [2] calculations of subquadratic second order Dehn functions for various groups were carried out. In this paper we obtain estimates for upper and lower bounds of second order Dehn functions of $H N N$-extensions, and use these to exhibit an infinite number of different superquadratic second order Dehn functions. At the end of the paper several open questions concerning second order Dehn functions of groups are discussed.
\end{abstract}

1991 Mathematics subject classification (Amer. Math. Soc.): primary 20E06, 20F05, 20F06; secondary 57M05, 57M07.

\section{Introduction}

Second (and higher) order Dehn functions have been studied in $[2,3,10,11,13,15,17]$. In particular, in [2] exact calculations of some (subquadratic) second order Dehn functions $\delta_{G}^{(2)}$ for various groups $G$ were carried out. For any positive integer $r$ examples were given of groups with second order Dehn function

$$
n \mapsto n^{2-1 / r} \text {. }
$$

Also, examples were given of groups with second order Dehn function

$$
n \mapsto \frac{n^{2}}{\log n}
$$

(C) 1999 Australian Mathematical Society 0263-6115/99 \$A2.00+0.00 
Recent work in [7], coupled with results in [2, Section 6] show that for any positive integers $p \geq q$ there is a group with second order Dehn function

$$
n \mapsto n^{2-1 / \rho}
$$

where $\rho=2 \log _{2}(2 p / q)$; in particular the set

$$
\left\{\alpha: \alpha \in\left[\frac{3}{2}, 2\right], n \mapsto n^{\alpha} \text { is a second order Dehn function }\right\}
$$

is dense in $[3 / 2,2]([7$, Theorem $\mathrm{C}])$.

In this paper we obtain estimates for upper and lower bounds of second order Dehn functions of $H N N$-extensions (Theorem 1 and Theorem 2). (We remark that similar estimates for general graphs of groups can be obtained.) We then obtain estimates for certain special $H N N$-extensions (Theorem 3), and use these to exhibit an infinite number of different superquadratic second order Dehn functions. For any positive integer $r$ we give an example (Example 1) of a group $G_{r}$ with $\delta_{G_{r}}^{(2)}$ lying between the functions

$$
n \mapsto n^{r / 2+1}, \quad n \mapsto n^{r+1} .
$$

Also, we give an example (Example 2) of a group $G_{\infty}$ with $\delta_{G_{\infty}}^{(2)}$ lying between the functions

$$
n \mapsto e^{\sqrt{n}}, \quad n \mapsto e^{n}
$$

At the end of the paper we raise some open questions concerning second order Dehn functions.

We refer the reader to [2, Section 2] (see also [3, 15, 17]) for information concerning second order Dehn functions, and for all unexplained notations. However, as suggested by the referee, we include here some of the main ideas for the readers convenience.

Let $\mathscr{P}=\langle\mathbf{x} ; \mathbf{r}\rangle$ be a finite group presentation. The group $G(\mathscr{P})$ defined by $\mathscr{P}$ is the quotient of the free group $F(\mathbf{x})$ on $\mathbf{x}$ by the normal closure of the elements of $F(\mathbf{x})$ represented by the words in $\mathbf{r}$. If $W$ is a word on $\mathbf{x}$ then the element of $G(\mathscr{P})$ represented by $W$ will be denoted by $\bar{W}$, and the word length of $W$ will be denoted by $L(W)$. Usually we will blur the distinction between a word and the element of $F(\mathbf{x})$ it represents.

Diagrams, or their duals, pictures, over presentations are a well-established concept. In this paper we will use pictures and adopt [6] as our main reference (see also [14]).

If $W$ is a word representing the identity of $G(\mathscr{P})$ then the area $A(W)$ of $W$ (with respect to $\mathbf{r}$ ) is the minimum area (that is, the number of discs) of any picture over $\mathscr{P}$ with boundary label $W$. The first order Dehn function $\delta_{\mathscr{G}}^{(1)}$ is defined by

$$
\delta_{\mathscr{P}}^{(1)}(n)=\max \{A(W) ; \bar{W}=1, L(W) \leq n\} .
$$


As is well-known (see [1] for example) if $\mathscr{Q}$ is another finite presentation with $G(\mathscr{P}) \cong$ $G(\mathscr{Q})$, then for a certain standard equivalence $\sim$ of functions (see below) we have $\delta_{\mathscr{g}}^{(1)} \sim \delta_{\mathscr{Q}}^{(1)}$. Thus up to $\sim$-equivalence, for a finitely presented group $G$ we have the (well-defined) first order Dehn function $\delta_{G}^{(1)}$ of $G$.

The equivalence $\sim$ is defined as follows. For two increasing functions

$$
f, g: \mathbb{N} \rightarrow \mathbb{R}^{+}
$$

write $f \preceq g$ if there is a constant $a>0$ such that $f(n) \leq a g(a n)+a n(n \in \mathbb{N})$. Then $f \sim g$ if $f \preceq g$ and $g \preceq f$.

There are certain operations (bridge moves, insertions or deletions of folding pairs, insertions or deletions of floating circles) which can be performed on (spherical) pictures (see [6, Section 1.2]), and two spherical pictures are said to be equivalent if one can be obtained from the other by a finite number of these operations. We write $\langle\mathbb{P}\rangle_{\mathscr{G}}$ (or simply $\langle\mathbb{P}\rangle$ ) for the equivalence class of the spherical picture $\mathbb{P}$. These equivalence classes form a left $\mathbb{Z} G(\mathscr{P})$-module (the second homotopy module of $\mathscr{P}$, denoted $\pi_{2}(\mathscr{P})$ ) under the addition

$$
\left\langle\mathbb{P}_{1}\right\rangle+\left\langle\mathbb{P}_{2}\right\rangle=\left\langle\mathbb{P}_{1}+\mathbb{P}_{2}\right\rangle
$$

(where $\mathbb{P}_{1}+\mathbb{P}_{2}$ is the spherical picture obtained by putting $\mathbb{P}_{1}$ next to $\mathbb{P}_{2}$ ), with $G(\mathscr{P})$-action

$$
\bar{W} \cdot\langle\mathbb{P}\rangle=\langle W \cdot \mathbb{P}\rangle
$$

(where $W \cdot \mathbb{P}$ is the picture obtained from $\mathbb{P}$ by surrounding it with a collection of concentric closed arcs with total label $W$-see [6, Figure V.6.(b)]).

Let $\mathbf{X}$ be a set of generators of the module $\pi_{2}(\mathscr{P})$. Then if $\mathbb{P}$ is a spherical picture we define the volume $V_{\mathbf{X}}(\langle\mathbb{P}\rangle)$ of $\langle\mathbb{P}\rangle$ with respect to $\mathbf{X}$ to be the least value of $m$ over all expressions

$$
\sum_{i=1}^{m} \varepsilon_{i} g_{i} \zeta_{i}
$$

$\left(\varepsilon_{i}= \pm 1, g_{i} \in G(\mathscr{P}), \zeta_{i} \in \mathbf{X}, i=1,2, \ldots, m\right)$ equal to $\langle\mathbb{P}\rangle$. We then define the second order Dehn function $\delta_{\mathscr{P}, \mathrm{X}}^{(2)}$ by

$$
\delta_{\mathscr{P}, \mathbf{X}}^{(2)}(n)=\max \left\{V_{\mathbf{X}}(\langle\mathbb{P}\rangle): A(\mathbb{P}) \leq n\right\} \quad(n=1,2, \ldots) .
$$

For a group $G$ of type $F_{3}$ (that is, a group given by a finite presentation for which $\pi_{2}$ is finitely generated) this is a group invariant up to -equivalence [3] (see also [15, 17]), which we write as $\delta_{G}^{(2)}$-the second order Dehn function of $G$. 


\section{Preliminaries}

Consider an $H N N$-extension

$$
G=\left\langle H, t ; t^{-1} K t \stackrel{\psi}{=} \hat{K}\right\rangle .
$$

Here $K, \hat{K}$ are subgroups of the group $H$, and

$$
\psi: K \rightarrow \hat{K}
$$

is an isomorphism. We will assume that $H$ is of type $F_{3}$ and $K, \hat{K}$ are finitely presented.

Choose a finite presentation $\mathscr{P}_{H}=\langle\mathbf{x} ; \mathbf{r}\rangle$ for $H$. Let $\mathbf{a}=\left\{a_{y}: y \in \mathbf{y}\right\}, \hat{\mathbf{a}}=$ $\left\{\hat{a}_{y}: y \in \mathbf{y}\right\}$ be finite sets of words on $\mathbf{x}$ representing generators of $K, \hat{K}$ respectively, where $\hat{a}_{y}$ represents the image under $\psi$ of the element of $K$ represented by $a_{y}$. Let $F=F(\mathbf{y})$ be the free group on $\mathbf{y}$, and let $B$ be the kernel of the epimorphism $F \rightarrow K$ induced by the mapping $y \mapsto a_{y}(y \in \mathbf{y})$. Let $\mathbf{s}$ be a finite set of words whose normal closure in $F$ is $B$. Then

$$
\mathscr{P}_{K}=\langle\mathbf{y} ; \mathbf{s}\rangle
$$

is a presentation of $K$.

For $W$ a word on $\mathbf{y}$, say $W=y_{1}^{\varepsilon_{1}} y_{2}^{\varepsilon_{2}} \cdots y_{n}^{\varepsilon_{n}}\left(y_{i} \in \mathbf{y}, \varepsilon_{i}= \pm 1,1 \leq i \leq n\right)$, we let $W(\mathbf{a})$ (respectively, $W(\hat{\mathbf{a}})$ ) denote the word on $\mathbf{x}$ obtained by replacing each $y_{i}$ by $a_{y_{i}}$ (respectively, $\hat{a}_{y_{i}}$ ). For $y \in \mathbf{y}$ let $Q_{y}$ denote the word $a_{y} t \hat{a}_{y}^{-1} t^{-1}$ and let $\mathbf{q}=\left\{Q_{y}: y \in \mathbf{y}\right\}$. Then a presentation for $G$ is given by

$$
\mathscr{P}=\mathscr{P}_{G}=\langle\mathbf{x}, t ; \mathbf{r}, \mathbf{q}\rangle \text {. }
$$

For $S \in \mathbf{s}$ we let $\mathbb{S}, \hat{\mathbb{S}}$ be pictures over $\mathscr{P}_{H}$ with (clockwise) boundary labels $S(\mathbf{a}), S(\hat{\mathbf{a}})$ respectively, and we set

$$
c=\max \{A(\mathbb{S}), A(\hat{S}): S \in \mathbf{s}\} .
$$

The abelianization $B^{a b}=B / B^{\prime}$ (written additively) of $B$ is a left $\mathbb{Z} K$-module (called the relation module of $\mathscr{P}_{K}$ ) under the action

$$
a_{y} \cdot W B^{\prime}=y W y^{-1} B^{\prime} \quad(y \in \mathbf{y}, W \in B) .
$$

This module is generated by the elements $S B^{\prime}$ ( $S \in \mathrm{s}$ ). Given $W \in B$ we can thus write $W B^{\prime}$ as a sum of elements $\pm k S B^{\prime}(k \in K, S \in \mathbf{s})$. We define $A^{a b}(W)$ to be the minimum number of terms in any such sum equal to $W B^{\prime}$. This is the 'abelianized area', considered in [5]. In general, $A^{a b}(W) \leq A(W)$. 
Let $\mathbb{D}$ be a picture over $\mathscr{P}_{K}$ with discs $\Delta_{1}, \ldots, \Delta_{m}$ labelled clockwise by $S_{1}^{\varepsilon_{1}}, \ldots$, $S_{m}^{\varepsilon_{m}}$ respectively $\left(S_{i} \in \mathbf{s}, \varepsilon_{i}= \pm 1,1 \leq i \leq m\right)$. We let $\mathbb{D}(\mathbf{a})$ (respectively, $\mathbb{D}(\hat{\mathbf{a}})$ ) denote the picture over $\mathscr{P}_{H}$ obtained from $\mathbb{D}$ by replacing the disc $\Delta_{i}(1 \leq i \leq m)$ by the picture $\varepsilon_{i} \Im_{i}$ (respectively, $\varepsilon_{i} \hat{\mathbb{S}}_{i}$ ), and for each $y \in \mathbf{y}$ replacing all arcs labelled $y$ by a sequence of parallel arcs with total label $a_{y}$ (respectively $\hat{a}_{y}$ ).

We let $\mathbb{P}_{\mathbb{D}}$ denote the spherical picture over $\mathscr{P}$ obtained from $\mathbb{D}(\mathbf{a}), \mathbb{D}(\hat{\mathbf{a}})$, as depicted in Figure 1, and we let $\xi_{\mathbb{D}}$ denote the element of $\pi_{2}(\mathscr{P})$ represented by $\mathbb{P}_{\mathbb{D}}$. When $\mathbb{D}$ consists of a single disc labelled $S \in \mathbf{s}$ then we write $\mathbb{P}_{S}$ (respectively $\xi_{S}$ ) instead of $\mathbb{P}_{\mathbb{D}}$ (respectively $\xi_{\mathbb{D}}$ ).

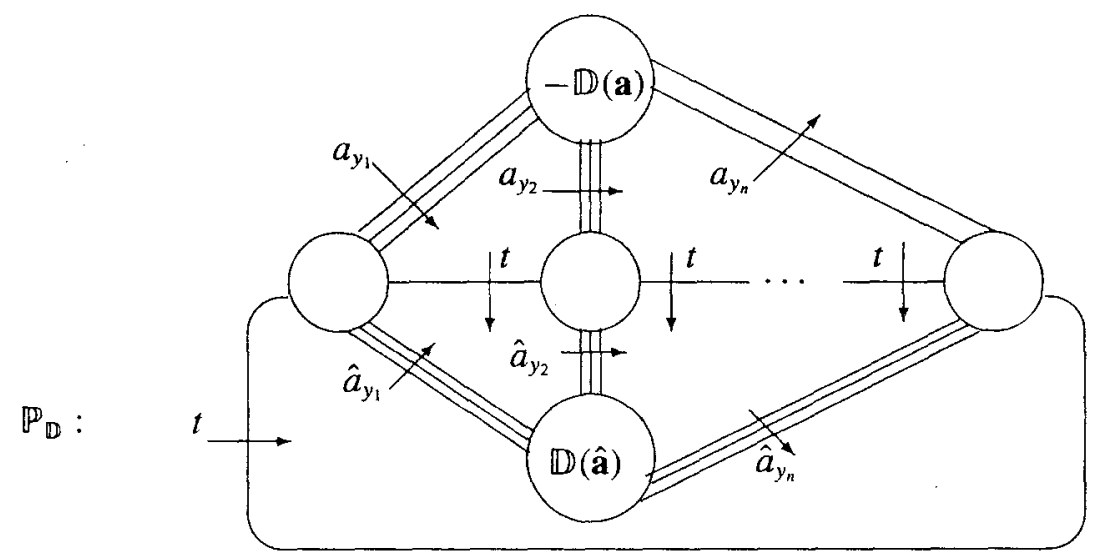

FiguRE 1.

Let $W$ be the label of $\mathbb{D}$. Choose a spray (see [14] for the definition) $\left(\gamma_{1}, \ldots, \gamma_{m}\right)$ for $\mathbb{D}$ and let $U_{i}$ be the label on $\gamma_{i}(i=1, \ldots, m)$. Then $W$ is freely equal to

$$
\prod_{i=1}^{m} U_{i} S_{i}^{\varepsilon_{i}} U_{i}^{-1}
$$

It follows that in the relation module $B^{a b}$ we have

$$
W B^{\prime}=\sum_{i=1}^{m} \varepsilon_{i} \bar{U}_{i} S_{i} B^{\prime}
$$

LEMMA 1. If $\mathscr{P}_{K}$ is aspherical and if for each $S \in \mathrm{s}$ there is no cancellation in the sum

$$
\sum_{S_{i}=S} \varepsilon_{i} \bar{U}_{i}
$$

then $A^{a b}(W)=m$. 
PROOF. If $\mathscr{P}_{K}$ is aspherical then $B^{a b}$ is freely generated as a $\mathbb{Z} K$-module by the elements $S B^{\prime}(S \in \mathbf{s})$. Thus, as an abelian group $B^{a b}$ is generated freely by the elements $k S B^{\prime}(k \in K, S \in \mathbf{s})$, and the result is then immediate.

$$
\text { LEMMA 2. } \xi_{\mathbb{D}}=\sum_{i=1}^{m} \varepsilon_{i} \overline{U_{i}(\mathbf{a})} \xi_{s_{i}} \text {. }
$$

The proof is similar to the proof of Lemma 5.1 of [2] and is left to the reader.

\section{Generators of the second homotopy module and upper bound for volume}

Let $\mathbb{B}_{1}, \ldots, \mathbb{B}_{r}$ be a collection of spherical pictures over $\mathscr{P}_{H}$ representing a set $\mathbf{X}_{H}$ of generators of $\pi_{2}\left(\mathscr{P}_{H}\right)$. Then each $\mathbb{B}_{i}$ also represents an element of $\pi_{2}(\mathscr{P})$. We let $\mathbf{X}$ denote the set of elements of $\pi_{2}(\mathscr{P})$ represented by $\mathbb{B}_{1}, \ldots, \mathbb{B}_{r}$, together with the elements $\xi_{S}(S \in \mathbf{s})$.

THEOREM 1. The set $\mathbf{X}$ generates $\pi_{2}(\mathscr{P})$, and if $\mathbb{P}$ is a spherical picture over $\mathscr{P}$ with $n$ discs, of which $l$ are $\mathbf{q}-$ discs, then

$$
V_{\mathbf{X}}(\langle\mathbb{P}\rangle) \leq \bar{\delta}_{\mathscr{P}_{H}, \mathbf{X}_{H}}^{(2)}\left(2 c \bar{\delta}_{\mathscr{P}_{K}}^{(1)}(l)+(n-l)\right)+\bar{\delta}_{\mathscr{P}_{K}}^{(1)}(l)
$$

and hence

$$
\delta_{G}^{(2)}(n) \preceq \bar{\delta}_{\mathscr{P}_{H}, \mathbf{X}_{H}}^{(2)}\left(2 c \bar{\delta}_{\mathscr{P}_{K}}^{(1)}(n)+n\right)+\bar{\delta}_{\mathscr{P}_{K}}^{(1)}(n) .
$$

(Here, as usual, for a function $\delta, \bar{\delta}$ denotes the subnegative closure, see [2, Section 2$]$.)

PROOF. Let $\mathbb{P}$ be a spherical picture over $\mathscr{P}$. A non-trivial $t$-circle (outward or inward directed) in $\mathbb{P}$ consists of a collection of $\mathbf{q}$-discs $\Theta_{1}, \ldots, \Theta_{k}(k>0)$ and a collection of $t$-arcs $\alpha_{1}, \ldots, \alpha_{k}$ where $\alpha_{i}$ joins $\Theta_{i-1}$ to $\Theta_{i}$ (subscripts $\bmod k$ ). We can assume that the numbering of the discs is such that they are encountered in the order $\Theta_{1}, \Theta_{2}, \ldots, \Theta_{k}$ where we read clockwise around the $t$-circle starting at $\Theta_{1}$. Suppose $\Theta_{i}$ is labelled by $Q_{y_{i}}^{\varepsilon_{i}}\left(y_{i} \in \mathbf{y}, \varepsilon_{i}= \pm 1\right)$. Then the label on the $t$-circle is the word $y_{1}^{\varepsilon_{1}} y_{2}^{\varepsilon_{2}} \cdots y_{k}^{\varepsilon_{k}}$. We also allow a trivial $t$-circle consisting of a single closed $t$-arc. The label on a trivial $t$-circle is the empty word. A $t$-circle $C$ is minimal if there is no $t$-circle contained in the region enclosed by $C$.

The proof will be by induction on the number $k$ of $t$-circles in $\mathbb{P}$.

If $k=0$ then $\mathbb{P}$ is a picture over $\mathscr{P}_{H}$, so $\langle\mathbb{P}\rangle_{\mathscr{P}_{H}}$ can be expressed as a sum of at most $\bar{\delta}_{\mathscr{P}_{H}, \mathbf{X}_{H}}^{(2)}(n)$ elements of $\pm H \mathbf{X}_{H}$. Thus $\langle\mathbb{P}\rangle$ can be expressed as a sum of at most $\vec{\delta}_{\mathscr{P}_{H}, \mathbf{X}_{H}}^{(2)}(n)$ elements of $\pm G \mathbf{X}$. 


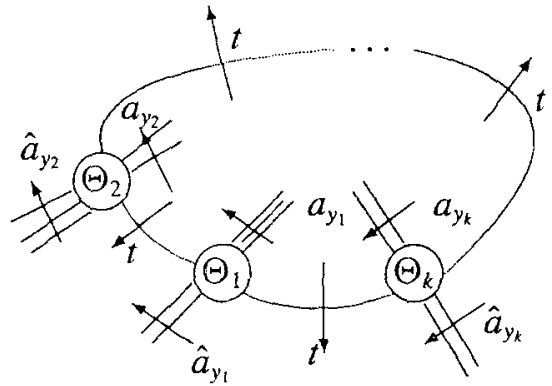

outward directed

$t$-circle

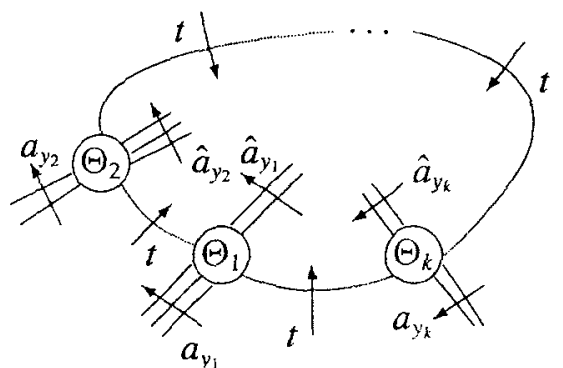

inward directed

$t$-circle

Figure 2.

Suppose $k>0$ and let $C$ be a minimal $t$-circle of $\mathbb{P}$ with label $W$. Let us first assume that $C$ is inward directed. Let $\mathbb{A}_{0}$ be the subpicture of $\mathbb{P}$ lying inside the area enclosed by $C$, and let $\mathbb{A}_{1}$ be the geometric configuration obtained by deleting $\mathbb{A}_{0}$ and $C$ from $\mathbb{P}$. Let $\mathbb{D}_{W}$ be a picture over $\mathscr{P}_{K}$ with boundary label $W$ and having $A(W)$ discs. Then $\mathbb{A}_{0}$ and $-\mathbb{D}_{W}(\hat{\mathbf{a}})$ can be combined to form a spherical picture $\mathbb{P}_{H}$ over $\mathscr{P}_{H}$, and $\mathbb{A}_{1}$ and $\mathbb{D}_{W}(\mathbf{a})$ can be combined to form a spherical picture $\mathbb{P}^{\prime}$ over $\mathscr{P}$. We then have for some word $U$ (see Figure 3)

$$
\langle\mathbb{P}\rangle=\left\langle\mathbb{P}^{\prime}\right\rangle+\bar{U}\left(\xi_{\mathbb{D}_{w}}+\bar{t}\left\langle\mathbb{P}_{H}\right\rangle\right)
$$

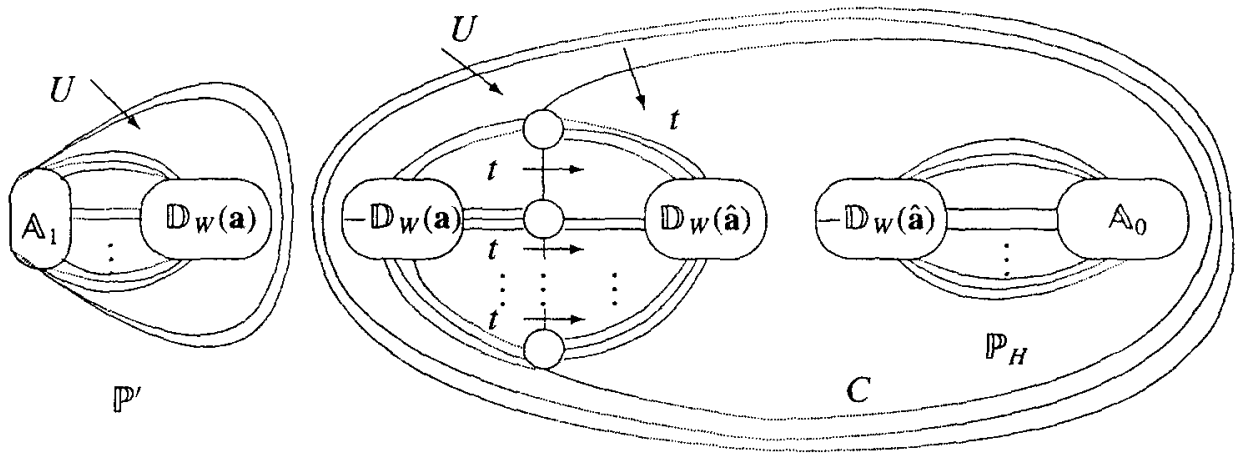

FIGURE 3.

Now $\mathbb{P}^{\prime}$ has fewer $t$-circles than $\mathbb{P}$. Moreover, the area of $\mathbb{P}^{\prime}$ is at most

$$
n-L(W)-A\left(\mathbb{A}_{0}\right)+c A(W)
$$


and the number of $\mathbf{q}$-discs in $\mathbb{P}^{\prime}$ is $l-L(W)$. Thus by inductive hypothesis $\left\langle\mathbb{P}^{\prime}\right\rangle$ can be expressed as a sum of at most

$$
\bar{\delta}_{\mathscr{P}_{H}, \mathbf{X}_{H}}^{(2)}\left(2 c \bar{\delta}_{\mathscr{P}_{K}}^{(1)}(l-L(W))+n-l-A\left(\mathbb{A}_{0}\right)+c A(W)\right)+\bar{\delta}_{\mathscr{P}_{K}}^{(1)}(l-L(W))
$$

elements of $\pm G \mathbf{X}$.

By Lemma $2, \xi_{\mathbb{D}_{W}}$ (and hence $\bar{U} \xi_{\mathbb{D}_{W}}$ ) can be expressed as a sum of $A(W)$ elements of $\pm G \mathbf{X}$, so $V_{\mathbf{X}}\left(\xi_{\mathbb{D}_{w}}\right)$ is at most

$$
\bar{\delta}_{\mathscr{P}_{K}}^{(1)}(L(W))
$$

Finally, since $\mathbb{P}_{H}$ is a spherical picture over $\mathscr{P}_{H}$, and since $A\left(\mathbb{P}_{H}\right) \leq A\left(\mathbb{A}_{0}\right)+c A(W)$, we have that $\left\langle\mathbb{P}_{H}\right\rangle$ (and hence $\bar{U} \bar{t}\left\langle\mathbb{P}_{H}\right\rangle$ ) can be expressed as a sum of at most

$$
\bar{\delta}_{\mathscr{P}_{H}, \mathbf{X}_{H}}^{(2)}\left(A\left(\mathbb{A}_{0}\right)+c A(W)\right)
$$

elements of $\pm G \mathbf{X}$.

Adding (3), (4), (5) and using the fact that $A\left(\mathbb{D}_{W}\right) \leq \bar{\delta}_{\mathfrak{P}_{k}}^{(1)}(L(W))$, we find that $\langle\mathbb{P}\rangle$ can be expressed as a sum of at most

$$
\bar{\delta}_{\mathscr{P}_{H}, \mathbf{x}_{H}}^{(2)}\left(2 c \bar{\delta}_{\mathscr{P}_{K}}^{(1)}(l)+(n-l)\right)+\bar{\delta}_{\mathscr{P}_{K}}^{(1)}(l)
$$

elements of $\pm G \mathbf{X}$, as required.

If $C$ is outward directed then a similar argument applies except now (2) becomes

$$
\langle\mathbb{P}\rangle=\left\langle\mathbb{P}^{\prime}\right\rangle-\bar{U} \bar{t}^{-1} \xi_{\mathbb{D}_{w}}+\bar{U} \bar{t}^{-1}\left\langle\mathbb{P}_{H}\right\rangle,
$$

where $\mathbb{P}_{H}$ is the result of combining $\mathbb{A}_{0}$ and $-\mathbb{D}_{W}(\mathbf{a})$, and $\mathbb{P}^{\prime}$ is the result of combining $\mathbb{A}_{1}$ and $\mathbb{D}_{W}(\hat{\mathbf{a}})$.

REMARK. Theorem 1 can easily be extended to multiple extensions of $H$ (where we allow several pairs of isomorphic subgroups of $H$ ). More generally, Theorem 1 can be extended to graphs of groups. Let $\Gamma$ be a finite connected directed graph with vertex set $\mathbf{v}$ and directed edge set $\mathbf{e}$. Suppose that for each vertex $v \in \mathbf{v}$ we have a group $H_{v}$ of type $F_{3}$, and for each directed edge $e$ running from vertex $u$ to vertex $v$ say, we have isomorphic finitely presented subgroups $K_{e} \leq H_{u}, \hat{K}_{e} \leq H_{v}$. Let $G$ be the corresponding fundamental group of this graph of groups. Then we get an upper bound for $\delta_{G}^{(2)}$ like that at the end of Theorem 1, with $\delta_{\mathscr{P}_{H}, \mathbf{x}_{H}}^{(2)}, \delta_{\mathscr{P}_{K}}^{(1)}$ replaced by

$$
\max \left\{\delta_{\mathscr{P}_{H_{v}}, \mathbf{x}_{H_{v}}}^{(2)}: v \in \mathbf{v}\right\}, \quad \max \left\{\delta_{\mathscr{P}_{K_{e}}}^{(1)}: e \in \mathbf{e}\right\}
$$

respectively. 


\section{Lower bound for volume}

Let $L$ be the submodule of $\pi_{2}(\mathscr{P})$ generated by the elements $\left\langle\mathbb{B}_{1}\right\rangle, \ldots,\left\langle\mathbb{B}_{r}\right\rangle$. Let $\mu$ be the standard embedding (see [2, Section 2]) of $\pi_{2}(\mathscr{P})$ into the free left $\mathbb{Z} G$-module with basis $e_{R}(R \in \mathbf{r}), e_{y}(y \in \mathbf{y})$ in one-to-one correspondence with the defining relators of $\mathscr{P}$. Since $\mu(L) \subseteq \bigoplus_{R \in \mathrm{r}} \mathbb{Z} G e_{R}$, we get an induced homomorphism

$$
\mu^{*}: \frac{\pi_{2}(\mathscr{P})}{L} \longrightarrow \bigoplus_{y \in \mathrm{y}} \mathbb{Z} G e_{y} .
$$

We have the Magnus embedding $\kappa$ (see [12, page 199]) of the relation module $B^{a b}$ of $\mathscr{P}_{K}$ into the free left $\mathbb{Z} K$-module $\bigoplus_{y \in y} \mathbb{Z} K e_{y}$. By applying the exact functor $\mathbb{Z} G \bigotimes_{\mathbb{Z} K}$-we then obtain an embedding

$$
\tilde{\kappa}: \mathbb{Z} G \bigotimes_{\mathbb{Z} K} B^{a b} \stackrel{1 \otimes \kappa}{\longrightarrow} \mathbb{Z} G \bigotimes_{\mathbb{Z} K}\left(\bigoplus_{y \in \mathbf{y}} \mathbb{Z} K e_{y}\right) \cong \bigoplus_{y \in \mathrm{y}} \mathbb{Z} G e_{y}
$$

and we have the commutative diagram

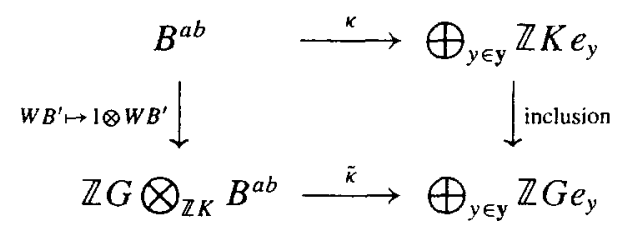

Now $\pi_{2}(\mathscr{P}) / L$ is generated by the elements $\xi_{S}+L(S \in \mathrm{s})$, and $\mathbb{Z} G \bigotimes_{\mathbb{Z} K} B^{a b}$ is generated by the elements $\beta_{S}=1 \otimes S B^{\prime}(S \in \mathrm{s})$. Since

$$
\mu^{*}\left(\xi_{S}+L\right)=\tilde{\kappa}\left(\beta_{S}\right) \quad(S \in \mathbf{s})
$$

we have that $\operatorname{Im} \mu^{*}=\operatorname{Im} \tilde{\kappa}$, so we get a surjective homomorphism

$$
\theta: \pi_{2}(\mathscr{P}) \stackrel{\text { nat }}{\longrightarrow} \frac{\pi_{2}(\mathscr{P})}{L} \stackrel{\tilde{x}^{-1} \mu^{*}}{\longrightarrow} \mathbb{Z} G \bigotimes_{\mathbb{Z} K} B^{a b} .
$$

Let $W$ be any word on $\mathbf{y}$ which defines an element of $B$. Consider a minimal expression

$$
\sum_{j=1}^{n} \varepsilon_{j} g_{j} \beta_{S_{j}}
$$

$\left(\varepsilon_{j}= \pm 1, g_{j} \in G, S_{j} \in \mathbf{s}\right.$ for $\left.j=1, \ldots, n\right)$ for $1 \otimes W B^{\prime}$ as a sum of elements from the set $\left\{ \pm g \beta_{S}: g \in G, S \in \mathbf{s}\right\}$. Clearly $n \leq A^{a b}(W)$. We want to show that in fact 
equality holds. We can suppose that for some $0 \leq l \leq n, g_{j} \in K$ if and only if $j \leq l$. Now $\tilde{\kappa}\left(1 \otimes W B^{\prime}\right)$ and $\tilde{\kappa}\left(\beta_{S}\right)(S \in \mathrm{s})$ belong to $\bigoplus_{y \in \mathbf{y}} \mathbb{Z} K e_{y}$ and so we deduce that

$$
\tilde{\kappa}\left(\sum_{j>l} \varepsilon_{j} g_{j} \beta_{S_{j}}\right)=0
$$

Hence, by the injectivity of $\tilde{\kappa}$ and the minimality of (6), $n=l$, that is, each $g_{j} \in K$. Thus we have

$$
\kappa\left(W B^{\prime}\right)=\tilde{\kappa}\left(1 \otimes W B^{\prime}\right)=\tilde{\kappa}\left(\sum_{j=1}^{n} \varepsilon_{j} g_{j} \beta_{S_{j}}\right)=\kappa\left(\sum_{j=1}^{n} \varepsilon_{j} g_{j} S_{j} B^{\prime}\right) .
$$

So (using the injectivity of $\kappa$ ), we deduce that $n \geq A^{a b}(W)$, as required.

Let $\mathbb{E}, \hat{\mathbb{E}}$ be any pictures over $\mathscr{P}_{H}$ with boundary labels $W(\mathbf{a}), W(\hat{\mathbf{a}})$ respectively. Then we can form a spherical picture as in Figure 1 with $\mathbb{D}(\mathbf{a}), \mathbb{D}(\hat{\mathbf{a}})$ replaced by $\mathbb{E}$, $\hat{\mathbb{E}}$. For simplicity we denote the element of $\pi_{2}(\mathscr{P})$ represented by this picture by $\zeta_{W}$ (though note that $\zeta_{W}$ depends on the choice of $\mathbb{E}, \hat{\mathbb{E}}$ ).

LEMMA 3. $V_{\mathbf{X}}\left(\zeta_{W}\right) \geq A^{a b}(W)$.

PROOF. Suppose we have an expression for $\zeta=\zeta_{W}$ as a sum of $V_{\mathbf{X}}(\zeta)$ terms from $\pm G \mathbf{X}$. Applying $\theta$ to this expression we will get an expression for $\theta(\zeta)$ as a sum of at most $V_{\mathbf{X}}(\zeta)$ terms from $\left\{ \pm g \beta_{S}: g \in G, S \in \mathbf{s}\right\}$. But $\theta(\zeta)=1 \otimes W B^{\prime}$ and so from our discussion above

$$
V_{\mathbf{X}}(\zeta) \geq A^{a b}(W)
$$

THEOREM 2. Let $f: \mathbb{N} \rightarrow \mathbb{N}, h:[1, \infty) \rightarrow[1, \infty)$ be functions with $h$ strictly increasing. Suppose there are words $W_{i}(i \in \mathbb{N})$ on $\mathbf{y}$ with:

(a) $L\left(W_{i}\right) \leq h(i)$

(b) $A\left(W_{i}(\mathbf{a})\right), A\left(W_{i}(\hat{\mathbf{a}})\right) \leq h(i)$;

(c) $A^{a b}\left(W_{i}\right) \geq f(i)$

for all $i$. Then

$$
\delta_{G}^{(2)}(n) \succeq f\left(\left[h^{-1}(n)\right]\right)
$$

(where [] denotes the integral part).

In the special case when $K=\hat{K}$ and $\psi$ is the identity, if we replace (a) by

(a') $l(i) L\left(W_{i}\right) \leq h(i)$,

where $l(i)$ is a function of the natural number $i$, we obtain

$$
\delta_{G}^{(2)}(n) \succeq l\left(\left[h^{-1}(n)\right]\right) f\left(\left[h^{-1}(n)\right]\right) .
$$


PROOF. For each $i$ we may choose $\mathbb{E}_{i}, \hat{\mathbb{E}}_{i}$ each to have area at most $h(i)$ (by (b)), and then $A\left(\zeta_{W_{i}}\right) \leq 3 h(i)$ (using (a)).

Now let $n \in \mathbb{N}$, and let $i=\left[h^{-1}(n)\right]$. Then $A\left(\zeta_{W_{i}}\right) \leq 3 n$, and by Lemma 3 and (c),

$$
V_{\mathbf{X}}\left(\zeta_{W_{i}}\right) \geq A^{a b}\left(W_{i}\right) \geq f\left(\left[h^{-1}(n)\right]\right) .
$$

Hence,

$$
\delta_{\mathscr{P}, \mathrm{X}}^{(2)}(3 n) \geq f\left(\left[h^{-1}(n)\right]\right)
$$

For the second part, first note that if

$$
\zeta_{W}^{(l)}=\left(1+\bar{t}+\cdots+\bar{t}^{l-1}\right) \zeta_{W}, \quad l=1,2, \ldots,
$$

then since powers of $\bar{t}$ all lie in different cosets modulo $K$, we can modify the argument leading to Lemma 3 to show that

$$
V_{\mathbf{X}}\left(\zeta_{W}^{(l)}\right) \geq l A^{a b}(W)
$$

Now in the special case when $K=\hat{K}$ and $\psi$ is the identity, $\zeta_{W}^{(l)}$ can be represented by a small picture, built up by using $-\mathbb{E}, \mathbb{E}$ and $l$ concentric $t$-circles (similar to Figure 13 in [2]), so that

$$
A\left(\zeta_{W}^{(l)}\right) \leq 2 A(W(\mathbf{a}))+l L(W)
$$

Thus, for this special case, if we replace condition (a) by ( $\left.\mathrm{a}^{\prime}\right)$ and modify the argument above we get

$$
\delta_{\mathscr{P}, \mathrm{X}}^{(2)}(3 n) \geq l\left(\left[h^{-1}(n)\right]\right) f\left(\left[h^{-1}(n)\right]\right)
$$

\section{Application}

Let $F$ be a finitely generated free group with basis $\mathbf{x}$, and let $\phi$ be a positive automorphism of $F$ (that is $\phi(x)$ is a positive word on $\mathbf{x}$ for all $x \in \mathbf{x}$ ). Form the semi-direct products $K=F \rtimes\langle s, \bar{s}\rangle, A=F \rtimes\langle u\rangle$ with $s, \bar{s}, u$ all acting via $\phi$. Let $H=A \times\langle v, \bar{v}\rangle$. There is an embedding of $K$ into $H$ given by

$$
F \stackrel{i d}{\longrightarrow} F, s \mapsto u v, \bar{s} \mapsto u \bar{v},
$$

and we can form the $H N N$-extension

$$
G=\left\langle H, t ; t^{-1} K t=K\right\rangle,
$$


which has presentation

$$
\begin{aligned}
\mathscr{P}= & \left\langle\mathbf{x}, u, v, \bar{v}, t ; u^{-1} x u=\phi(x),[v, x],[\bar{v}, x],[x, t](x \in \mathbf{x}),\right. \\
& {[v, u],[\bar{v}, u],[u v, t],[u \bar{v}, t]\rangle . }
\end{aligned}
$$

Let $Z_{n}(n=1,2, \ldots)$ be a sequence of positive words on $\mathbf{x}$ such that $L\left(Z_{n}\right) \leq \alpha n$ for some constant $\alpha$ (independent of $n$ ), and let

$$
f(n)=\sum_{i=0}^{n-1} L\left(\phi^{i}\left(Z_{n}\right)\right) .
$$

THEOREM 3. $\sqrt{n} f([\sqrt{n}]) \preceq \delta_{G}^{(2)}(n) \preceq \bar{\delta}_{K}^{(1)}(n) \quad(n=1,2,3, \ldots)$.

PROOF. By making the substitution $s=u v, \bar{s}=u \bar{v}$ in the presentation $\mathscr{P}$ above, and performing Tietze transformations we find that

$$
G \cong K \rtimes\langle u, t\rangle
$$

where $u$ acts via the automorphism $\hat{\phi}$ of $K$ defined by

$$
F \stackrel{\phi}{\longrightarrow} F, s \mapsto s, \bar{s} \mapsto \bar{s},
$$

and $t$ acts via the identity automorphism. We can thus regard $G$ as a (multiple) $H N N$ extension where the base-group and associated subgroups are all equal to $K$, and can then apply Theorem 1 (for multiple $H N N$-extensions). Since $K$ has the aspherical presentation

$$
\mathscr{P}_{K}=\left\langle\mathbf{x}, s, \bar{s} ; s^{-1} x s=\phi(x), \bar{s}^{-1} x \bar{s}=\phi(x)(x \in \mathbf{x})\right\rangle
$$

we have that $\bar{\delta}_{\mathcal{P}_{\kappa}, \emptyset}^{(2)}$ is the zero function. So we deduce from Theorem 1 that

$$
\delta_{G}^{(2)}(n) \preceq \bar{\delta}_{K}^{(1)}(n) .
$$

To establish the lower bound we revert to the original presentation (7) of $G$, and use the second part of Theorem 2 with $f$ as above, $h(\lambda)=(4+2 \alpha) \lambda^{2}$ and $l(n)=n$. Note that

$$
\mathscr{P}_{H}=\left\langle\mathbf{x}, u, v, \bar{v} ; u^{-1} x u=\phi(x),[v, x],[\bar{v}, x](x \in \mathbf{x}),[v, u],[\bar{v}, u]\right\rangle,
$$

$\mathscr{P}_{K}$ is as in (8), and

$$
a_{x}=\hat{a}_{x}=x(x \in \mathbf{x}), a_{s}=\hat{a}_{s}=u v, a_{\bar{s}}=\hat{a}_{\bar{s}}=u \bar{v} .
$$




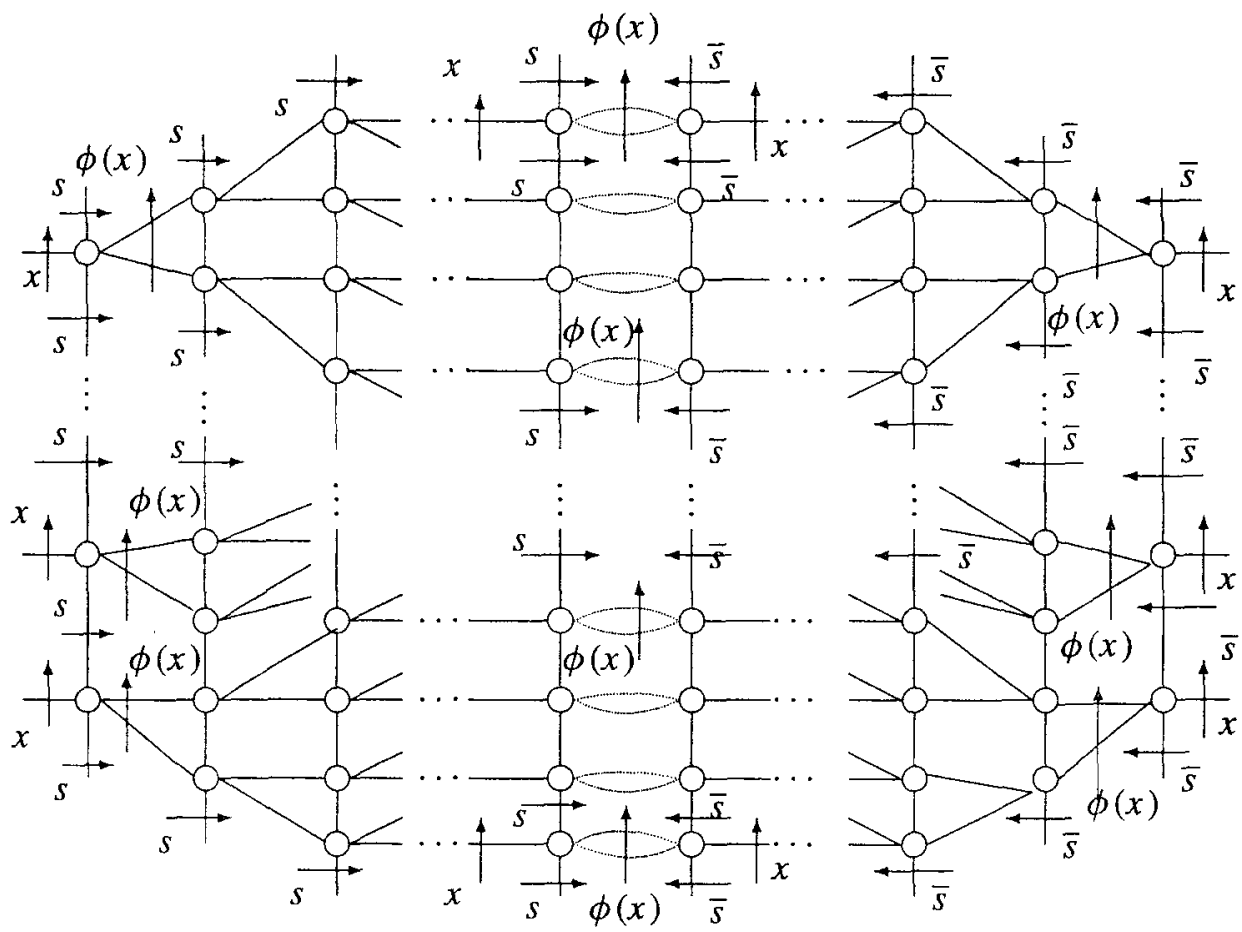

FIGURE 4.

Let $W_{n}=s^{-n} Z_{n} s^{n} \bar{s}^{-n} Z_{n}^{-1} \bar{s}^{n} \quad(n=1,2, \ldots)$. Then

$$
L\left(W_{n}\right) \leq(4+2 \alpha) n \text {. }
$$

We have the picture $\mathbb{D}_{n}$ with boundary label $W_{n}$ over $\mathscr{P}_{K}$ as in Figure 4 . Since $\mathscr{P}_{K}$ is aspherical it follows from Lemma 1 that

$$
A^{a b}\left(W_{n}\right)=2 f(n) .
$$

We also have the picture $\mathbb{E}_{n}$ with boundary label $W_{n}(\mathbf{a})$ over $\mathscr{P}_{H}$ as in Figure 5. This picture has area $2 n^{2}+2 n\left(L\left(Z_{n}\right)+1\right)$, so

$$
A\left(W_{n}(\mathbf{a})\right) \leq 2(2+\alpha) n^{2} .
$$

EXAMPLE 1. Let $F$ be free on $x_{1}, x_{2}, \ldots, x_{r}$ and let $\phi$ be defined by

$$
x_{i} \mapsto x_{i} x_{i+1}(1 \leq i<r), \quad x_{r} \mapsto x_{r} .
$$

Let $Z_{n}=x_{1}^{n}$. Then

$$
f(n)=n\left[\left(\begin{array}{l}
n \\
1
\end{array}\right)+\left(\begin{array}{l}
n \\
2
\end{array}\right)+\cdots+\left(\begin{array}{l}
n \\
r
\end{array}\right)\right],
$$




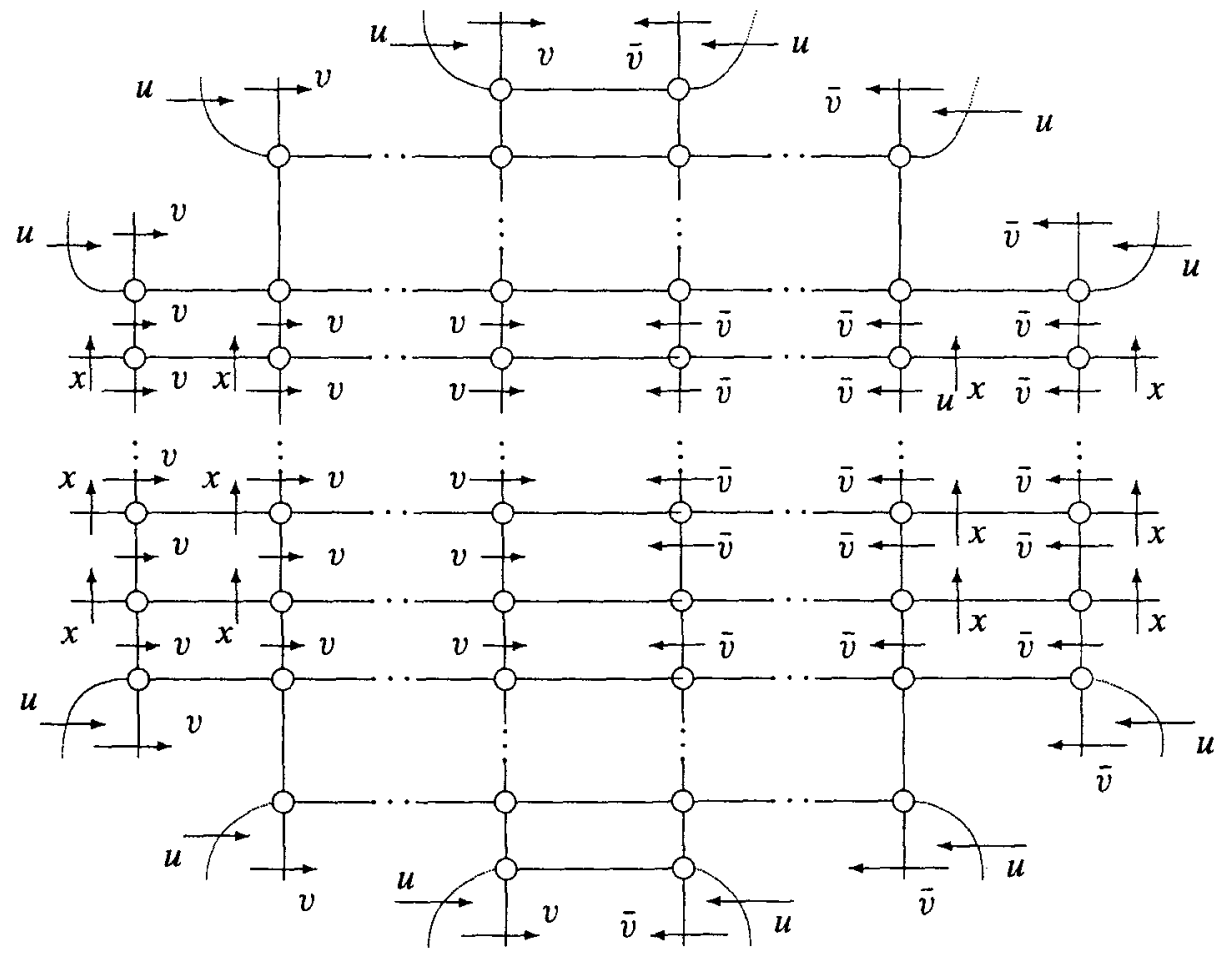

FIGURE 5.

and so it follows that

$$
[\sqrt{n}] f([\sqrt{n}]) \sim n^{(r+2) / 2} .
$$

On the other hand, it has been shown by Bridson [8] that for this situation

$$
\delta_{K}^{(1)}(n) \sim n^{r+1}\left(\sim \bar{\delta}_{K}^{(1)}(n)\right) .
$$

Thus we deduce that

$$
n^{(r+2) / 2} \preceq \delta_{G}^{(2)}(n) \preceq n^{r+1}
$$

EXAMPLE 2. Let $F$ be free of rank 3 on $a, b, c$ and let $\phi$ be defined by

$$
a \rightarrow c, b \rightarrow a c, c \rightarrow b c .
$$

Let $Z_{n}=c$ for all $n$. Then as shown in [2] (see page 26)

$$
f(n) \sim e^{n} .
$$


Also, it is shown in $[4$, Example 2] that

$$
\delta_{K}^{(1)}(n) \sim e^{n}\left(\sim \bar{\delta}_{K}^{(1)}(n)\right) .
$$

Thus we have

$$
e^{\sqrt{n}} \preceq \delta_{G}^{(2)}(n) \preceq e^{n}
$$

\section{Open questions}

The results of this paper combined with those in $[2,7]$ mentioned in the introduction give some idea of the behaviour of second order Dehn functions of groups, but the overall picture is still far from clear. We mention the following open questions.

(i) Obtain some exact calculations of superquadratic second order Dehn functions. In particular:

(ii) Is there a group with exactly quadratic second order Dehn function? (Added in proof: Yes-Martin Bridson, to appear.) For the group $G_{2}$ mentioned in the introduction, is $\delta^{(2)}$ quadratic?

(iii) Describe the spectrum of second order Dehn functions, that is the set of $\alpha$ for which

$$
n \mapsto n^{\alpha}
$$

is a second order Dehn function.

(iv) Characterize groups with linear second order Dehn function. (Clearly groups with aspherical presentations are in this class. It is shown in [2] that hyperbolic groups are also in this class.)

(v) How small can $\delta^{(2)}$ be? The split extension of $\mathbb{Z}^{2}$ by $\mathbb{Z}$ with the generator of $\mathbb{Z}$ acting via the automorphism

$$
\left(\begin{array}{ll}
2 & 1 \\
1 & 1
\end{array}\right)
$$

was shown in [16] to have second order Dehn function bounded below by

$$
n \mapsto n \log n
$$

It follows from [9] that this lower bound is in fact exact (we thank C. Pittet for pointing this out to us). This is the smallest non-linear second order Dehn function we know of. 
(vi) How large can $\delta^{(2)}$ be? For the group $G_{\infty}$ mentioned in the introduction, $\delta^{(2)}$ is more or less exponential. However, no examples above exponential are known. The techniques of this paper could probably be used to obtain such examples if the following question could be addressed.

(vii) Can one embed a finitely presented group with large (eg. $n \mapsto 2^{2^{n}}$ ) first order Dehn function into a group of type $F_{3}$ with small (for example polynomial) first order Dehn function?

In connection with (vi) and (vii) see also [13, Question 1 and Question 2].

In [13] it is shown that the second order Dehn function of a group is subrecursive (see also [17]). There then arises the following question.

(viii) Is every second order Dehn function equivalent to a recursive function?

\section{Acknowledgment}

We thank Stuart McGlashan for his comments on an initial draft of this paper. We also thank the referee for a careful reading of the paper and for the helpful comments.

\section{References}

[1] J. M. Alonso, 'Inégalités isopérimétriques et quasi-isometries', C. R. Acad. Sci. Paris Sér. I Math. 311 (1990), 761-764.

[2] J. M. Alonso, W. A. Bogley, R. M. Burton, S. J. Pride and X. Wang, 'Second order Dehn functions of groups', Quart. J. Math. Oxford Ser. (2) 49 (1998), 1-30.

[3] J. M. Alonso, X. Wang and S. J. Pride, 'Higher dimensional isoperimetric (or Dehn) functions of groups', J. Group Theory 2 (1999), 81-112.

[4] G. Baumslag, M. R. Bridson, C. F. Miller III and H. Short, 'Finitely presented subgroups of automatic groups and their isoperimetric functions', J. London Math. Soc. 56 (1997), 292-304.

[5] G. Baumslag, C. F. Miller III and H. Short, 'Isoperimetric inequalities and the homology of groups', Invent. Math. 113 (1993), 531-560.

[6] W. A. Bogley and S. J. Pride, 'Calculating generators of $\pi_{2}$ ', in: Two-dimensional homotopy theory and combinatorial group theory (eds. W. Metzler, C. Hog-Angeloni and A. Sieradski), London Mathematical Society Lecture Note Series 197 (Cambridge Univ. Press, Cambridge, 1993) pp. 157-188.

[7] N. Brady and M. R. Bridson, 'There is only one gap in the isoperimetric spectrum', Technical report (1998).

[8] M. R. Bridson, 'Polynomial isoperimetric inequalities and the length of asynchronously automatic structures', Technical report (University of Oxford, 1996).

[9] T. Coulhon and L. Saloff-Coste, 'Isoperimetrie pour les groupes et les varietes', Rev. Mat. Iberoamericana 9 (1998), 293-314.

[10] D. B. A. Epstein, J. W. Cannon, D. F. Holt, S. V. Levy, M. S. Paterson and W. P. Thurston, Word processing in groups (Bartlett and Jones, Boston, 1992). 
[11] M. Gromov, 'Asymptotic invariants of infinite groups', in: Geometric group theory (eds. G. Niblo and M. Roller), London Math. Soc. Lecture Note Series 182 (OUP, 1993).

[12] P. J. Hilton and U. Stammbach, A course in homological algebra, GTM 4 (Springer, New York, 1971).

[13] P. Papasoglu, 'Some remarks on isodiametric and isoperimetric inequalities', Technical report (1998).

[14] S. J. Pride, 'Identities among relations of group presentations', in: Group theory from a geometric viewpoint (Trieste, 1990) (eds. E. Ghys, A. Haefliger and A. Verjovsky) (World Sci. Publ., Singapore, 1991) pp. 687-717.

[15] X. Wang, 'Mappings of groups and quasi-retractions', J. Group Theory, to appear.

[16] - Second order Dehn functions of groups and monoids ( $\mathrm{Ph} . \mathrm{D}$. Thesis, University of Glasgow, 1996).

[17] X. Wang and S. J. Pride, 'Second order Dehn functions of groups and monoids', Internat. J. Alg. Comp., to appear.

Department of Mathematics

Shenzhen University

Shenzhen City 518060

Guangdong

P. R. China

e-mail: wangxf@szu.edu.cn
Department of Mathematics The University of Glasgow University Gardens Glasgow G12 8QW UK e-mail: sjp@maths.gla.ac.uk 